\title{
Allein gegen den Rest der Welt - REPRÄSENTATIONEN VON MÄNNLICHKEITEN IM DEUTSCHROCK BEI WESTERNHAGEN UND DEN BÖHSEN ONKELZ
}

\section{Dietmar Elflein}

Ausgangspunkt für die folgenden Überlegungen ist die Beobachtung, dass eine in der angelsächsischen Populärkultur des 20. Jahrhunderts häufig anzutreffende rebellische Männerfigur erst mit erheblicher Verspätung - d.h. Mitte der 1970er Jahre - in der deutschen Popularmusik bzw. spezifischer im Deutschrock heimisch wird. Die für diesen Aneignungsprozess notwendige Übersetzungsleistung verändert mindestens Teile der Männerfigur sukzessive und passt sie so an die veränderten Gegebenheiten der deutschen Popularmusik an.

Um diese Transformation näher einzugrenzen, wird in der Folge erst der verwendete Begriff von Männlichkeit definiert und die gewählte Methodik dargelegt. Im Anschluss muss geklärt werden, was unter Deutschrock verstanden werden soll, die Auswahl der Beispiele begründet werden und schließlich die Transformation dieser Männerfigur anhand der gewählten Beispiele nachgezeichnet werden. Es handelt sich bei den hier verhandelten Repräsentationen von Männlichkeit um medial vermittelte Images, die ausschließlich in medialen Veröffentlichungnen bzw. als Performance auf der Konzertbühne existieren. Aussagen über die hinter der jeweiligen Männerfigur stehenden Künstlerpersönlichkeiten sind weder intendiert noch gewollt.

Die angelsächsischen Wurzeln der infrage stehenden Männerfigur sollen aus Platzgründen nur angerissen werden: Der betreffende Mann ist (sub-) proletarischen Ursprungs, von heller Hautfarbe und besitzt eine rebellische Grundhaltung, meist gekoppelt mit Klassenbewusstsein. Er weiß zwar, dass er auf lange Sicht keine Chance hat, sich auf dem nur vermeintlich freien Markt durchzusetzen, versucht es aber trotzdem (immer wieder). Seine rebellische Haltung macht inn zum Glücksritter, der geboren ist, um zu 
verlieren, aber lebt, um trotzdem zu gewinnen. ${ }^{1}$ Auf dem Siegertreppchen erwartet inn die als sozialer Aufstieg getarnte Teilhabe - zumindest am Recht auf Konsum. Er sucht die individuelle Freiheit z.B. auf dem Motorrad und wird im Film von Marlon Brando in The Wild One (Benedek 1953) beispielhaft verkörpert. Bezüge zur sich als rebellisch verstehenden, aber gleichzeitig konservativen Bikerkultur (vgl. exemplarisch Barger 2003) finden sich insgesamt häufig. Gefährliche Heldenfiguren der US-amerikanischen Westernmythologie wie Jesse James, Billy the Kid und Han Solo ${ }^{2}$ sind ihm zumindest verwandt. Bezogen auf populäre Musik spiegeln Rockabilly und die Images des frühen Elvis Presley, von Jerry Lee Lewis und insbesondere Gene Vincent diese Männerfiguren. Easy Rider (Hopper 1968) transportiert den Mann filmisch in die von der Mittelklasse geprägte Gegenkultur der Beatniks und Hippies. Musikalisch machen die Rolling Stones die Verbindung des infrage stehenden Rebellen zur Arbeiterklasse brüchig. Dagegen findet man ihn weiterhin sowohl bei den Teds, den Rockern, den Mods als auch den Skins, die in ihrer britischen und australischen Ausprägung Rebellion mit Klassenbewusstsein koppeln. Er spielt in Bands wie AC/DC, Rose Tattoo ${ }^{3}$ und Motörhead Rock und Bluesrock. Progressives ist inm zumindest musikalisch fremd.

\section{Männlichkeiten in der populären Musik}

Trotz des in der Literatur konstatierten Aufschwungs der Männerforschung seit Mitte der 1990er Jahre (vgl. Poole 2012: 10-12; Fenske 2012: 11f.; Horlacher 2013: 28-30) steht die Auseinandersetzung mit Männlichkeiten in der populären Musik noch am Anfang; eine eigenständige Theoriebildung existiert de facto nicht (vgl. Heesch/Losleben 2012, Gerards/Loeser/Losleben $2013)^{4}$. Dies gilt umso mehr, wenn man sich mit der skizzierten weißen und

1 »Born to lose, live to win« ist einer der offizieller Slogans der englischen Band Motörhead (vgl. http://www.imotorhead.com, Zugriff am 1.5.2014).

2 Kevin Burns' Dokumentarfilm Star Wars: Die Geschichte einer Saga (2010) definiert die Star Wars-Saga als einen Science Fiction-Western.

3 Die Gewerkschaftshymne »Union Man « (2002) zeigt exemplarisch das vorhandene Klassenbewusstsein von australischen Rock'n'Roll Outlaw[s] (Rose Tattoo 1980), wo »Nice Boys « (1980) keinen Rock' $n$ ' Roll spielen.

4 In Heesch/Losleben (2012) beschäftigt sich nur ein, zudem nicht sonderlich aktueller, Artikel mit Männlichkeit in der populären Musik: Robert Walsers (2012) bereits 1993 erschienene Ausführungen zu Männlichkeit im Heavy Metal. In Gerards/Loeser/Losleben (2013) liegt zwar der Fokus der Beiträge auf populärer Musik, der Forschungsüberblick von Katrin Losleben (2013: 67f.) widmet der populären Musik trotzdem nur knapp zwei von 17 Seiten. 
heterosexuellen Männerfigur beschäftigt (vgl. der Überblick in Horlacher 2013: 37f.). In der Literaturwissenschaft hat ein derartiger Bezug zwar eine längere Tradition, meine Recherche förderte aber ebenfalls nicht viele, zumindest aber aktuellere Arbeiten zu Tage (vgl. Simmons 2008; Poole 2012; Fenske/Schuhen 2012).

Deswegen bildet R.W. (Raewyn) Connells (1995) Konzept der hegemonialen Männlichkeit auch für diesen Beitrag die Grundlage. Standard der Männerforschung in der Folge von Connell ist, dass nicht mehr von Männlichkeit an sich, sondern von unterschiedlich miteinander in Beziehung und Konkurrenz stehenden Männlichkeiten ausgegangen wird (vgl. beispielhaft Fenske/Schuhen 2012; Poole 2012: 12-14). Connell selbst unterscheidet hegemoniale, komplizenhafte, marginalisierte und untergeordnete Männlichkeiten. Die Einordnung nicht hegemonialer weißer heterosexueller Männlichkeiten in dieses Schema erscheint uneindeutig. Poole (2012: 14f.) verweist darauf, dass die notwendige Nuancierung von Männlichkeiten auch nonkonforme Männlichkeiten erlauben müsse, die gleichwohl gefährlich für die Gesellschaft als auch insbesondere andere Geschlechter sein können. Michael Meuser (2012) differenziert das Bild der hegemonialen Männlichkeit aus soziologischer Perspektive. Er behauptet Veränderungen der ursprünglich in der bürgerlichen Gesellschaft verankerten hegemonialen Männlichkeit, die in journalistischer Hinsicht unter dem Schlagwort »Krise der Männlichkeit « zusammengefasst werden können. Die flexibilisierte und deregulierte Arbeitswelt lässt die für Männlichkeit grundlegenden Parameter des Lebensarbeitsplatzes und der Rolle des Familienernährers zunehmend zur Ausnahme werden. Gleichzeitig ist paternalistisches Management nicht mehr überall gefragt. Die männlichen ernsten Spiele des Wettbewerbs gewinnen nach Meuser deshalb im Rahmen flacher Hierarchien an Schärfe. Die QuasiFeminisierung des Arbeitslebens führt, wiederum nach Meuser, zudem zur imaginären Stärkung der angegriffenen Männlichkeit mittels symbolischer Überzeichnung.

Dies und die Tatsache, dass ein Klassenunterschied zwischen den hier verhandelten und hegemonialen Männern besteht, schließt eine eindeutige Zuordnung nonkonformer Männlichkeiten zu komplizenhaften Männlichkeiten aus. Connell wiederum differenziert marginalisierte Männlichkeiten in erster Linie über Rasse und Migration sowie untergeordnete Männlichkeit über Homosexualität. Da in den marginalisierten Männlichkeiten damit eher ein Klassenbezug zu finden ist als in der untergeordneten Männlichkeit, soll in der Folge von marginalisierten Männlichkeiten ausgegangen werden. Arbeiten zu den hier verhandelten Männlichkeiten beschäftigen sich bisher vor allem mit rechtsextremen Männern (vgl. Claus/Lehnert/Müller 2010), gren- 
zen jedoch Aspekte einer Individualisierung aus dem rechtsextremen Männerbild aus (vgl. Virchow 2010: 42). Phänomene des White Trash respektive subproletarischer und bedrohter proletarischer Männlichkeit werden bisher kaum und wenn dann als Zerrbilder hegemonialer Männlichkeit (vgl. Poole 2012: 123-160; Projektgruppe Mannopoly 2012) verhandelt.

Die symbolische Überzeichnung angegriffener (hegemonialer) Männlichkeit sowie die Perspektive der marginalisierten Männlichkeiten sollen im Verlauf wieder aufgegriffen und zumindest im Ansatz am Gegenstand überprüft werden. Die infrage stehenden Männlichkeiten im Deutschrock können als kulturelle Repräsentationen einer imaginären Stärkung angegriffener Männlichkeiten begriffen werden.

Die angestrebte vergleichende Perspektive von Männerbildern im Deutschrock nutzt Elemente der gender-orientierten Narratologie (vgl. Fenske 2012: 21f.). Narrativität wird dabei als transmediale Kulturtechnik verstanden, die Geschlechterverhältnisse hervorbringt und stabilisiert (vgl. ebd.). "Männlichkeit bestünde demzufolge aus einer Serie kulturell geprägter scripts und den daraus jeweils unterschiedlich und individuell gebildeten Geschichten« (Erhardt 2005: 217; Hervorhebung im Original). Diese Geschichten bilden sich im hier vorliegenden Fall in den Songtexten ab, die deshalb in der Folge primär untersucht werden. Dabei muss mit Frith (2006), wie bei jeder Analyse von Songtexten, immer klar sein, dass der Analysegegenstand gesungen und nicht gelesen wird, es sich also im Sinne Bakhtins bei Songtexten eher um Äußerungen mit einer spezifischen »expressive intonation« (Bakhtin 1986: 90) als um Texte handelt.

\section{Deutschrock und die Auswahl der Beispiele}

Der Begriff Deutschrock meint in diesem Artikel zwei historisch abgegrenzte Phänomene: Erstens eine in der Mitte der 1970er Jahre in der BRD beginnende allmähliche Loslösung von dem zeitgenössisch verbreiteten Dogma, dass deutsche Texte ein inhaltlich zu begründender Sonderfall der Rockmusik seien, und zweitens werden seit Mitte der 2000er Jahre Bands in der Nachfolge der Böhsen Onkelz unter dieser Begrifflichkeit subsumiert.

Im Rahmen der Geschichte der populären Musik in Deutschland galt Deutsch lange Zeit als die Sprache des Schlagers und der Liedermacher. Rockmusik verschmolz dagegen als Import aus dem angelsächsisch dominierten Raum quasi mit der englischen Sprache. Deutsch sei vom klanglichen Ausdruck zu hart für Rockmusik, die Sprachmelodie sei ungeeignet, so lauteten verbreitete Argumente (vgl. exemplarisch Niemczyk 2006). Trotzdem 
datieren die ersten zaghaften und selten kommerziell erfolgreichen Versuche, die deutsche Sprache mit angelsächsisch geprägter Popmusik zu verbinden, bereits aus den frühen bis mittleren 1960er Jahren (vgl. Drafi Deutscher And His Magics 1965; Die Yankees 1965; V.A. 2000). Nichtsdestotrotz wird die Nürnberger Band Ihre Kinder wegen ihres 1969 erschienenen Debüts (Ihre Kinder 1969) in den meisten Nachschlagewerken als erste Rockband mit deutschen Texten bezeichnet. Sie wird genau wie die Westberliner Band Ton Steine Scherben häufig unter dem Schlagwort Politrock subsumiert beispielsweise im Lexikon der populären Musik (Wicke/Ziegenrücker/Ziegenrücker 2004: 442), in dem Politrock wiederum als Synonym für Rockmusik mit deutscher Sprache gilt. Deutsche Liedtexte begründen ihre Existenz in der bundesrepublikanischen Rockmusik also über sozialkritische und politisch links engagierte Inhalte. Ausbruchsversuche aus dieser engen inhaltlichen Klammer werden in der zweiten Hälfte der 1970er Jahre dann unter Deutschrock subsumiert, dabei bleibt der sozialkritische linke Gestus zumindest abgeschwächt erhalten. Musikalisch orientiert sich der beginnende Deutschrock stark an US-amerikanischen Vorbildern, namentlich an Chuck Berry und einem von Chess Records geprägten Bluesrock- und Rock 'n'Roll-Verständnis bzw. an den Musikern und Songs, die so ähnlich bereits die Beatles und die Rolling Stones beflügelt hatten. Der lyrische Aufbruch des Deutschrock setzt also musikalisch auf Bewährtes, Experimente wie im Krautrock sind nicht gefragt. Vorbilder finden die Deutschrocker auch im Mundartrock, der jedoch mehrheitlich eine Untergruppe des Politrock bleibt. Hier hört man bei einigen Vertretern bereits eine starke BluesrockAffinität, z.B. bei den bayerischen Sparifankal (Bayern Rock 1976), die zum Teil aus Moderatoren der Jugendsendung des Bayerischen Rundfunks - Club 16 bzw. ab 1979 Zündfunk - bestehen, und kommerziell erfolgreich bei den Kölnern BAP. Damit grenzt man sich (un-)willentlich voneinander ab: auf der einen Seite die Experimentatoren und Elektroniker mit ihrer Suche nach einem nicht angelsächsisch geprägten künstlerischen Ausdruck (vgl. Whalley 2009), auf der anderen Seite die Deutschrocker und in der Mitte der Rest: von Progressive und Hard Rock sowie Fusion bis hin zu Folk(rock). ${ }^{5}$

5 Die Geschichte deutschsprachiger Rockmusik verläuft in der DDR anders, da englische Songtexte zu Eigenkompositionen nicht denkbar waren. Die Beatmusik der 1960er Jahre ist in der DDR deshalb zu einem höheren Prozentsatz instrumental, ab Anfang der 1970er Jahre etabliert sich dann eine deutschsprachige Rockmusik, deren Texte mehrheitlich nicht von den Bands selbst, sondern von professionellen Lyrikern verfasst werden und die vor der Veröffentlichung staatliche Zensurinstanzen durchlaufen muss. Sozialkritische Inhalte sind in der Rockmusik der DDR deshalb meist sehr verklausuliert anzutreffen. Die Geschichte der Rockmusik in der DDR ist in den Arbeiten von u.a. Michael Rauhut (1993, 
Als paradigmatisch für Deutschrock gilt retrospektiv im Allgemeinen das Schaffen von Udo Lindenberg. ${ }^{6}$ Dieser wird hier aus mehreren Gründen ignoriert: Zum einem arbeitet Lindenberg mit dem Panikorchester an der Wiederbelebung von Revueideen aus der Zeit vor dem Nationalsozialismus. Er ging »ständig mit ausgefallenen Ideen über Musik und ihre Präsentation schwanger« (Haring 1984: 77f.) und bezeichnete sich selbst laut Günter Ehnert (1979: 152) als »Musik-Artist«. Dementsprechend heterogen fällt auch sein musikalisches Schaffen aus, das nur in Teilen als Rockmusik bezeichnet werden kann (vgl. Haring 1984: 78) und von Dixieland über Boogie Woogie und Schlager bis zum Rock reicht. Zum anderen doppelt sich das Revuekonzept in den lyrischen Protagonisten der Songtexte, deren Namen gerne auch als Songtitel dienen (»Rudi Ratlos«, 1974; »Bodo Ballermann«, 1976 etc.). Diese Rollen verkörpert der Performer Lindenberg und zeigt sich damit in Bezug auf das performte Männerbild ähnlich chamäleonhaft wie in musikalischer Hinsicht. Den Männerfiguren von Lindenberg fehlt deshalb in weiten Teilen die hier im Fokus stehende (sub-)proletarische und angelsächsische Orientierung. ${ }^{7}$

Musiker aus dem Umfeld von Lindenbergs Panikorchester arbeiten jedoch in der Folge an der Einführung eines Bluesrock orientierten Deutschrock ohne offensiv sozialkritischen Impetus entscheidend mit, beispielsweise als Mitglieder der Bands von Peter Maffay ${ }^{8}$ und Marius Müller-Westernhagen (vgl. Kravetz/Drewing 2008: 74-77 sowie 112-142; Kübler 2005). Beide Bandleader verknappen mit Hilfe dieser Musiker nicht nur ihre musikalischen Einflüsse, sondern auch das männliche Rollenangebot auf ein einziges genau ausformuliertes, das im Folgenden am Beispiel von Marius Müller-Westernhagen thematisiert werden soll.

Spätestens nach der Wiedervereinigung verliert die lyrisch und performativ geprägte Deutschrock-Definition ihren Sinn (vgl. Niemczyk 2006).

1996) sowie Peter Wicke und Lothar Müller (1996) besser wissenschaftlich aufgearbeitet als die bundesrepublikanische Rockmusik.

6 Die Betonung der Wichtigkeit von Lindenberg schreibt sich seit den 1970er Jahren fort (vgl. Ehnert 1979: 150-153, Haring 1984: 77-81). Aktuell gilt Lindenberg z.B. Medien wie Der Spiegel, Musikexpress und Der Freitag als Erfinder des Deutschrock (vgl. http://www.spiegel.de/kultur/musik/deutschrock-erfinder-l indenberg-ich-sehe-mich-als-kleinen-bruder-von-hermann-hesse-a-723147.html; http://www.musikexpress.de/artists/udo-lindenberg; http://www.freitag.de/ autoren/betrachter/eine-laudatio-auf-den-erfinder-des-deutschrock, Zugriff jeweils am 1.5.2014).

7 Eine wissenschaftliche Aufarbeitung von Lindenbergs Schaffen fehlt bisher leider sowohl in gendertheoretischer als auch musikwissenschaftlicher Hinsicht.

8 Peter Maffay gelingt mit Steppenwolf (1979) das erste Nummer Eins-Album, das dem Deutschrock zugerechnet werden kann. 
Songtexte, die Alltagsthemen in deutscher Sprache behandeln, sowie Bluesrock inspirierte Musik sind aufgrund der Auswirkungen der Neuen Deutschen Welle auf die Rockmusik der BRD und der rockmusikalischen Traditionen in der DDR kein hinreichendes Alleinstellungsmerkmal mehr. Die Bezeichnung Deutschrock taucht deshalb erst nach dem Millennium und insbesondere nach der Trennung der Böhsen Onkelz 2005 wieder verstärkt in den Medien auf. ${ }^{9}$ Sie meint nun Bands aus einer im weitesten Sinne musikalischen Punktradition, die sich lyrisch explizit von politisch links stehenden Positionen abgrenzen - häufig im Sinne einer sich als unpolitisch verstehenden neuen Mitte. Die neue alte Bezeichnung dient wiederum auch als Gegenbegriff zum zumindest teilweise sozialkritischen Ex-Punkrock der Ärzte und der Toten Hosen. Der neue Deutschrock wandert damit im Gegensatz zum alten nicht von der politisch linken, sondern von der politisch rechten Seite in Richtung Mitte. Das von den Böhsen Onkelz angebotene Männerbild wird deshalb im Anschluss auf Gemeinsamkeiten und Unterschiede zu dem von Westernhagen verkörperten Männerbild untersucht. Zunächst sollen beide jedoch kurz vorgestellt werden.

\section{Die Böhsen Onkelz}

Das visuelle Image der 1979 in Frankfurt/M. gegründeten Böhsen Onkelz ist anfangs stark an proletarisch geprägten englischen Jungenkulturen ausgerichtet. Das Cover des zweiten Albums Böse Menschen Böse Lieder (1985) zeigt die vier Bandmitglieder als Angehörige derartiger Jungenkulturen: Wir finden den Skin (Sänger Kevin Russell), den Ted (Gitarrist Matthias Röhr) und den proletarischen Punk (Bassist Stephan Weidner), auch als Streetpunk bezeichnet, der nicht am studentischen Art School-Teil von Punk orientiert ist (vgl. Frith/Horne 1987). Diese Jungenkulturen setzen auf Bewahrung traditionell männlicher Werte und nicht auf Modernisierung und Veränderung.

Der kommerzielle Erfolg der Böhsen Onkelz beginnt nach der Wiedervereinigung. 1992 erreicht das siebte Album der Band, Heilige Lieder, Platz fünf der deutschen Albumcharts. Dieser Durchbruch geht mit einer Veränderung des jungenkulturellen Bezugs einher. Auf dem Cover von Heilige Lieder (1992) sind die Haare der Bandmitglieder unterschiedlich lang gewachsen, nur Schlagzeuger Peter Schorowsky bildet eine visuelle Konstante. Das

9 Vgl. exemplarisch http://www.metal-hammer.de/magazin/features/article3353 36/deutschrock-special-von-broilers-bis-frei-wild.html; http://www.impact-ma ilorder.de/Freiwild_\&_die_Deutschrock_Szene_Angriff_der_Onkelz-Klone 84. html (Zugriff jeweils am 1.5.2014). 
Image ist damit nicht mehr an Repräsentationen unterschiedlicher und spezifischer Jungenkulturen ausgerichtet. Gleichzeitig bleiben Tätowierungen als Insignien der proletarischen Herkunft während der ganzen Karriere sehr wichtig für das Selbstverständnis der Band. Die von Böhsen Onkelz als Vorbilder benannten australischen Rose Tattoo (vgl. Weckmann 2010) tragen die proletarische Affinität zu Tätowierungen schon im Bandnamen.

Die Chartpräsenz von Heilige Lieder (1992) führt in Zusammenhang mit dem damals von der breiten Öffentlichkeit erstmals wahrgenommenen Erstarken des Neofaschismus im wiedervereinigten Deutschland zu einer anhaltenden Skandalisierung der Band als Aushängeschild einer rechtsextremen Ausprägung der Rockmusik - des sogenannten Rechtsrock (vgl. Hartsch 1997: 163-169; Röhr/Larmann 2006: 7-9). Die Böhsen Onkelz distanzieren sich zu diesem Zeitpunkt bereits seit mehreren Jahren von ihrer zum Teil rechtsradikalen Frühphase (vgl. Eberwein/Drexler 1987: 11-21; Matthesius 1992: 172-178). Konstitutiv für das Selbstverständnis der Band und damit auch die Inszenierung der Männlichkeit erscheint vielmehr die sich in den 1980ern aus der Fußballfanszene heraus entwickelnde Hooligan-Szene (vgl. Matthesius 1992: 171-190; Hartsch 1997).

Der kommerzielle Erfolg der Onkelz hält nach Heilige Lieder (1992) an. Die letzten vier Studioalben der Band erreichen alle Platz Eins der deutschen Charts, das zur Bandauflösung veranstaltete Open Air auf dem Lausitzring zieht 2005120.000 Besucher an. Spätestens im Anschluss an die Bandauflösung schießen Böhse Onkelz-Coverbands wie Pilze aus dem Boden (Stainless Steel, Die Glorreichen Halunken, E.I.N.S., Heilige Bruehder etc.). Diesen, wie auch Bands im Stile der Böhsen Onkelz (Kneipenterroristen, Kärbholz, Frei.Wild etc.), bietet das Festival »G.O.N.D [Größte OnkelzNacht Deutschlands] - den Onkelz sei ein Fest« seit 2006 eine Heimat und prägt so den neuen Deutschrock mit. Das Festival ist von Beginn an immer ausverkauft und seit 2009 auf 20.000 Besucher limitiert. ${ }^{10}$ Die Massenwirksamkeit der Onkelz erscheint damit bis in die Gegenwart ungebrochen. Seit den 1990er Jahren setzt die Band sukzessive ihre künstlerische und ökonomische Unabhängigkeit durch und besitzt sowohl ihre eigene Produktionsfirma als auch ihr eigenes Management. Dieses als Aktiengesellschaft organisierte Firmenkonglomerat verwaltet seither auch den Nachlass der Onkelz sowie die neuen Veröffentlichungen von Onkelz-Bassist und Texter Stephan Weidner als DER W. Am 30. Januar 2014 gab die Band offiziell ihre Reunion bekannt.

10 Vgl. http://www.gond.de und http://de.wikipedia.org/wiki/G.O.N.D. (Zugriff am 17.2.2014). 


\section{Marius Müller-Westernhagen}

Marius Müller-Westernhagen ist mit seinen Alben Nummer Vier bis Sechs ab 1978 konstitutiv für die erste Generation des Deutschrock. Alle drei Alben werden mindestens vergoldet. Stinker (1981), der Abschluss der Trilogie, erreicht mit Platz Fünf die höchste Notierung der drei Alben in den deutschen Charts. Ähnlich wie bei den Böhsen Onkelz folgt der kommerzielle Höhepunkt erst nach der Wiedervereinigung. Für den Anschub der Karriere Ende der 1970er ist es zumindest nicht abträglich, dass der Text von »Dicke« (auf Mit Pfefferminz bin ich dein Prinz, 1978) nach der Veröffentlichung als diskriminierend skandalisiert wird (vgl. Rossacher 2010).

Westernhagen erreicht seinen kommerziellen Durchbruch auch und vor allem über einen Imagewechsel. Er überträgt die Figur des proletarischen Kleinkriminellen Theo Gromberg, den er in zwei Filmen spielt, auf seine Musikkarriere. Die Figur Theo taucht erstmals in dem 1975 geschriebenen und zwei Jahre später gesendeten Fernsehfilm Aufforderung zum Tanz (Bringmann 1977) auf, 1978 erfolgt die Imageänderung des Musikers Westernhagen. Ab 1980 läuft schließlich der Film Theo gegen den Rest der Welt (Bringmann 1980) äußerst erfolgreich in den westdeutschen Lichtspielhäusern. ${ }^{11}$ Die Kinoauswertung setzt damit bereits auf der Bekanntheit von Westernhagen als Musiker auf. Interessanterweise wird dieser offensichtliche Zusammenhang in biografischen Veröffentlichungen von Westernhagen als purer Zufall dargestellt (vgl. Rossmann 2010'2). Das Cover von Mit Pfefferminz bin ich dein Prinz (1978) spielt mit seinen Assoziationen an Kneipe, Prostitution und Spielhölle mit dem Image des Theo und unterscheidet sich in der Stilisierung des Protagonisten stark vom Cover des Vorgängeralbums Ganz allein krieg ich's nicht hin (1977), das auf Sakko, Hemd und Schnauzbart setzt. Westernhagen zeichnet zudem laut Albumcredits für die Konzeption des Covers selbst verantwortlich, Fotograf Michael Ballhaus bestätigt dies im Interview (vgl. Rossacher 2010). Das von Westernhagen präsentierte Männerbild hat zwar nichts mit den Jungenkulturen zu tun, auf das sich die Böhsen Onkelz beziehen, betont jedoch ebenfalls proletarische Klischees

11 Der Film erhält 1982 eine Goldene Leinwand und hat damit in den ersten 18 Monaten nach Veröffentlichung mehr als drei Millionen Zuschauer und Zuschauerinnen gefunden.

12 Das dem Musiker Westernhagen gewidmete Kapitel »Müllermania « ignoriert die Bezüge zum filmischen Schaffen. Das Kapitel »Der Schauspieler « ist anfangs bewusst nicht chronologisch geordnet und stellt so den Zusammenhang zwischen Aufforderung zum Tanz, musikalischem Imagewechsel und Theo gegen den Rest der Welt als Zufall dar. 
wie das Goldkettchen sowie die körperlicher Arbeit entgegengesetzte Kleidungsfarbe weiß. ${ }^{13}$

\section{Repräsentationen von Männlichkeit bei Marius Müller-Westernhagen}

Sowohl die Böhsen Onkelz als auch »Theo Westernhagen ${ }^{14}$ repräsentieren proletarische Aussteigerfiguren. Im Gegensatz zur überwiegenden Mehrheit der Aussteigerfiguren, die den deutschen Film vor Aufforderung zum Tanz (Bringmann 1977) bevölkern (vgl. z.B. Klick 1974) ${ }^{15}$, ist die Figur Theo als positive Identifikationsfigur angelegt. ${ }^{16} \mathrm{Er}$ kämpft einen aussichtslosen Kampf um Anerkennung und sozialen Aufstieg, denn die Gesellschaft hält für inn keine attraktive Perspektive bereit. Theo verklärt die Alternative des Fernfahrerdaseins romantisch als Metapher für Freiheit und Selbstständigkeit und zieht sie einem geregelten Alltag und harter körperlicher Arbeit vor. Auf der Straße gibt es keinen Chef, sondern nur vermeintliche Geschäftspartner, denen der Mann fälschlicherweise auf Augenhöhe zu begegnen meint. Theo ist Egoist und Individualist, ein Möchtegern-Unternehmer seiner selbst und damit Vorbote einer postmodernen Männerfigur als pre-

13 Vgl. die TV-Auftritte von Westerhagen (z.B. http://www.youtube.com/watch? $\mathrm{v}=$ LHWXAeY1PgM, Zugriff am 17.2.2014) sowie das VHS-Video In Concert (1983), das in Ausschnitten ebenfalls auf YouTube zu finden ist (z.B. http://www. youtube.com/watch?v=npQHU635pek, Zugriff am 17.2.2014).

14 Martin Loesers (2013) eher hermeneutisch angelegte Analyse von Männlichkeitsinszenierungen bei Westernhagen und Grönemeyer ignoriert die Figur des Theo. Persona und Performer sind damit annähernd deckungsgleich. Dagegen geht mein Ansatz von einer grundsätzlichen Differenz zwischen Persona und Performer aus und nimmt Loesers Fazit zum infrage stehenden Karriereabschnitt von Westernhagen als Ausgangspunkt: Auf Mit Pfefferminz bin ich dein Prinz (1978) werden »keine Siegertypen, sondern durchweg eher als ambivalent und brüchig angesehene Konzeptionen von Männlichkeit präsentiert « (Loeser 2013: 125).

15 Der Titelsong »Celebration « für Roland Klicks Film Supermarkt (1974) wurde von Lindenberg geschrieben und von Westernhagen unter dem Pseudonym Marius West eingesungen (vgl. http://www.rolandklick.de/musik.html, Zugriff am 17.2.2014). Als Folge erhielt Westernhagen seinen ersten Plattenvertrag.

16 May Spils' Zur Sache, Schätzchen (1968) präsentiert zwar bereits eine positiv besetzte Aussteigerfigur, die jedoch eindeutig nicht proletarisch, als vielmehr bohemienhaft angelegt ist und deutliche Bezüge zur französischen Nouvelle Vague aufweist. Klaus Lemkes Paul (1974) zeigt dagegen bereits eine Nähe zu einem Punk-artigen No Future-Gestus, dem Theos Wille zum Aufstieg fehlt und deshalb seine Funktionalisierung als positive Identifikationsfigur schwer fällt. 
karisierter scheinselbstständiger Freiberufler. ${ }^{17}$ Die Aufhebung der Klassenidentifikation erscheint als einziger Ausweg aus der gefühlten Enge der proletarischen Zuschreibung. Westernhagen zitiert diese Haltung im Titelsong von Mit Pfefferminz bin ich dein Prinz (1978): "Glaubst Du an den lieben Gott oder an Guevara? Ich glaube an die Deutsche Bank, denn die zahlt aus in bar, aah!«

Sein Gegner ist das traditionelle paternalistische Management, das filmisch als Allianz aus Großbürgertum, Unternehmertum und organisierter Kriminalität inszeniert wird. Im Überlebenskampf muss Theo physische und psychische Niederlagen ertragen. Die resultierenden Schmerzen erträgt er, denn es geht ihm darum Träume zu leben, es geht um Momente eines richtigen Lebens im falschen, es geht im Einklang mit der musikalischen Neuorientierung um Sex, Drugs \& Rock'n'Roll, aber auch um eine depressive Grundstimmung, die von manischen Momenten unterbrochen wird.

Theo Westernhagen ist ein proletarisches Zerrbild hegemonialer Männlichkeit auf dem Weg in die komplizenhafte Männlichkeit, allerdings ohne Chance diese zu erlangen. Er betreibt aktiv seine Vereinzelung und Entwurzelung, während sein italienischer Sidekick Enno Goldini verzweifelt an proletarischen Werten wie Solidarität festzuhalten versucht, jedoch insbesondere von Theo nur ausgenutzt wird.

Frauen kommen bei Westernhagen zumindest in dieser Phase nur wahlweise als Sexobjekte oder unattraktiv gewordene Ehefrauen vor. Bei letzteren wird eine bessere Vergangenheit beweint, die Beziehung bzw. Ehe ist in der Gegenwart und Zukunft nur noch als Zwangsgemeinschaft vorstellbar:

»Ja, ich weiß, dass du mich liebst

und dass du mich nie betrügst.

Wen willst du auch schon verführen

mit den verheulten Augenlidern?

Ja, ich brauche meine Freunde,

junge Mädchen, die gern lachen.

In unserem Schlafzimmer aus Schleiflack

hab' ich mal an Mord gedacht.

Ja, ich weiß, mein Schatz,

dass wir mal glücklich waren.

Doch das Rezept haben wir verlegt «

(Marius Müller-Westernhagen: »Mein Schatz«, 1980).

17 Auch Böhse Onkelz-Bassist Weidner arbeitete Mitte der 1980er Jahre zeitweise als Kraftfahr-Subunternehmer und war so »wenigstens sein eigener Herr « (Hartsch 1997: 88). 
Während die Frauen sich in dieses Schicksal fügen sollen - »Der Junge auf dem weißen Pferd, der kommt nicht mehr « textet Westernhagen für das ebenfalls auf Sekt oder Selters (1980) enthaltene gleichnamige Stück -, bleibt für Männer die Option auszubrechen offen, da nur die Frau passiv und vergeblich auf Rettung durch den Märchenprinzen warten muss. Der Mann braucht dagegen seine Freunde und junge Mädchen, um sich lebendig zu fühlen; dass der »Schatz « das zu akzeptieren hat, steht außer Frage. Eine passive und leidende Männerfigur wird von Theo Westernhagen im zweiten Stück von Mit Pfefferminz bin ich dein Prinz deshalb wie folgt angegangen: »Klaus, nun wehr dich doch! Verdammt, du träumtest doch davon, dein eigener Herr zu sein!« (»Zieh dir bloß die Schuhe aus«, 1978). Die Musik spiegelt dabei den Bewusstseinszustand der verhandelten Figur: »Mein Schatz « bedient sich des Bo Diddley-Rhythmus und verweist damit auf eine der prägenden Inszenierungen afroamerikanischer Männlichkeit im Rock'n' Roll, während »Der Junge auf dem weißen Pferd« als Rock'n' Roll-Ballade im gemäßigten Tempo angelegt ist und »Zieh dir bloß die Schuhe aus « als Vorkriegsschlager mit leichten Jazzeinflüssen, um die unmännliche Rückständigkeit der beschriebenen Figur zu doppeln.

Eine der Empathie für die unterschiedlichsten männlichen Verlierer ähnliche Gefühlsregung bei anderen Geschlechtern empfindet der von Westernhagen inszenierte Mann nur bei Prostituierten (»Lulu«, 1981). Zwischen Mann und Prostituierter ist zudem erfüllter Sex und vielleicht sogar ein bisschen Liebe möglich, insbesondere wenn der Freier die erhaltene sexuelle Dienstleistung (vorerst) nicht bezahlen muss (»Sie hat meine Lunte gefunden«, 1980). ${ }^{18}$ Ansonsten betrachtet Theo Westernhagen Frauen als Trophäen. »Lady« (1980) thematisiert dabei offensiv den Klassenunterschied zwischen Jäger und Gejagter. Die Trophäenfrau verkörpert hier das männlich-proletarische Recht auf temporäre Freiheit von gesellschaftlichen Zwängen: »Und wenn ich auch nur Müller heiß, so bin ich doch am Leben« (»Lady«). Alle drei Stücke sind dementsprechend Uptempo-Bluesrock-Nummern.

Dass auch andere Frauen wider Erwarten Initiative ergreifen können, wissen die von Westernhagen in der Rolle des Theo verkörperten Männerfiguren, aber sie müssen zumindest so tun, als wäre es ihnen egal - das gebieten das Selbstwertgefühl und die männliche Peergroup.: »Dass du mich verlässt, das tut mir nicht weh« (»Daß du mich verläßt«, 1981) ertönt wiederum als Uptempo-Rock'n' Roll. »Hass mich oder lieb mich, es ist mir egal« (»Hass' mich oder lieb' mich «, 1980) ist an Folkblues-Vorbilder angelehnt. Während »Daß du mich verläßt« damit den Trotz zelebriert, weiß »Hass'

18 Dieses Geschlechterverhältnis könnte auch als Import aus Western-Filmen gedeutet werden. 
mich oder lieb' mich« (1980) auch musikalisch zu vermitteln, dass die Trennung eben nicht egal ist. Deswegen hilft Mann im Anschluss nur die Flucht in die Drogen, musikalisch von akustischen Gitarren im Fingerpicking-Stil untermalt: "Johnny Walker, du bist mein bester Freund. [...] Kein Mensch hört mir so gut zu wie du, Johnny, du lachst mich auch nie aus« (»Johnny W.«, 1978).

Der porträtierte Mann wird in der Konsequenz wahlweise erst zum Egoisten und dann zum Misanthropen oder umgekehrt. Auf jeden Fall ist die Welt nicht bereit, ihm den verdienten Respekt zu geben - wofür auch immer. Er ist grundsätzlich unzufrieden und dementsprechend auf dem Sprung. Bereits der erste Song des den Imagewandel einleitenden Albums Mit Pfefferminz bin ich dein Prinz (1978), »Mit 18«, thematisiert diese Unzufriedenheit mit dem jeweiligen Ist-Zustand, kann aber natürlich auch als einfache Ankündigung des Imagewechsels gelesen werden, wenn man dem lyrischen Ich zugesteht, wie Westernhagen eine Vergangenheit in Form von drei Alben zu haben:

»Ich möcht zurück auf die Straße, möcht wieder singen, nicht schön, sondern geil und laut.

Denn Gold find' man bekanntlich im Dreck.

Und Straßen sind aus Dreck gebaut «

(Marius Müller-Westernhagen: »Mit 18«, 1978).

In musikalischer Hinsicht macht »Mit 18 « über ein einleitendes Zwiegespräch von Mundharmonika und Stimme, das in einen Boogie mündet, den Imagewechsel hin zu einem klischierten Verständnis proletarischer Männlichkeit deutlich.

Dagegen findet sich die egoistische, individualisierte und nicht mehr in traditionellen politischen Zuschreibungen verortete Haltung der von Westernhagen in Anlehnung an Theo Gromberg erdachten Männerfiguren in lyrischer Hinsicht auch schon vor dem Imagewandel. Der »Träumer « (1977) aus dem Eröffnungsstück seines dritten Albums glaubt wider besseren Wissens an das Gute im Menschen. Er kann noch hoffen, dass die Parteipolitik erkennt, dass sie ihren andauernden Streit zu Gunsten eines größeren Ganzen, des Menschen im Allgemeinen und Besonderen, zurückstellt: »Rechte, Linke, Liberale erkennen auf 'ner Konferenz, dass sie sich stets gestritten haben, nur auf Kosten von uns Menschen« (»Träumer«, 1977). Die totalitaristischen Implikationen des hier vertretenen Weltbildes seien nur angemerkt, interessanter erscheint, dass es Westernhagen darum zu gehen scheint, den Albumtitel Ganz allein krieg ich's nicht hin (1977) von einer Bitte um Hilfe zu einer Ankündigung persönlicher Vorteilsnahme mutieren zu lassen: »Ich 
werde nach wie vor nur an mich denken und den Anhalter im Regen lass ich stehen« (»Träumer«, 1977).

Damit wird der Imagewandel, die offensive Repräsentation der OutlawMännlichkeit via Theo, mittels einer lyrischen Verengung und einer musikalischen Neuorientierung umgesetzt: Auf dem Weg von Ganz allein krieg ich's nicht hin (1977) zu Mit Pfefferminz bin ich dein Prinz (1978) wird die Instrumentierung der einzelnen Stücke auf eine Kernbesetzung mit zwei Gitarren, Bass und Schlagzeug reduziert - also eine Standard-Rock-Besetzung. Weitere Instrumente wie Tasteninstrumente und Saxophon sind im Rahmen des Albumklangs Zugabe und nicht mehr gleichberechtigt. Orchesterklänge verschwinden fast völlig. Damit einher geht eine allgemeine Ausdünnung des Klangbildes, die viel stärker eine Konzertsituation in einem kleinen Club simulieren will als ein große Bühne. Die Songstrukturen werden gleichfalls einfacher und stärker an Bluesformen angelehnt. Der Melodieumfang, der vorher gern den Stimmumfang von Westernhagen ausschöpft, wird ebenfalls verkleinert. Stattdessen orientiert sich der Stimmklang an Blues Shoutern und wird deshalb stilisiert und mit persönlichen klanglichen Manierismen angereichert. Beispielhaft bildet sich diese neue Stimme mit all ihren Möglichkeiten im oben bereits zitierten »Lady« (1980) ab, während »Träumer « (1977) auch musikalisch für den alten Westernhagen stehen kann.

Westernhagen inszeniert mittels der Figur des Theo das Zerrbild eines proletarischen Mannes, dessen Durchhaltevermögen im Alltag über den Exzess nach Feierabend funktioniert. Er betont dabei ausdrücklich, dass diese mit der Privatperson Marius Müller-Westernhagen nichts zu tun habe, vielmehr spiele er eine Rolle (vgl. Rossacher 2010). Im Gegensatz zu Lindenberg wechselt Westernhagen nicht die Rollen auf der Bühne, sondern die von ihm geschaffene Bühnenfigur interpretiert jeweils die unterschiedlichen lyrischen Ichs der Texte. Diese an Theo Gromberg angelehnte Bühnenfigur präsentiert eine fatalistische und individualistische Lösung für die Modernisierungen der Arbeitswelt und die Anforderungen, die damit auch auf Männer zukommen. Mann ist nur als Egoist überlebensfähig, Beziehungen jeglicher Art sind deshalb bloß als ökonomische und ökonomisierte denkbar. Solidarisch und zuverlässig sind nur die anderen, die noch an überkommene Werte wie Freundschaft und Solidarität glauben. Und so folgt aus dem Loch in der Tasche, durch das das Geld am Wochenende verschwindet, im letzten Song des Albums Stinker, das die von Theo inspirierte Trilogie abschließt: »Ich hab'n Loch in meinem Hirn und das kann man nicht mehr nähen. Und hab ich dir was versprochen, dann wirst du davon wohl nichts sehn « (»Ein Loch in der Tasche«, 1981). 


\section{Repräsentationen von Männlichkeit bei den Böhsen Onkelz}

Die Böhsen Onkelz treten im Gegensatz zu Westernhagen als Gruppe auf. Ihre Männerhorde präsentiert sich am Anfang ihrer Karriere als rebellisch, proletarisch und konservativ und verwirklicht sich in Alkohol- und Gewaltexzessen, die musikalisch affirmativ als Punkrock mit einfachen, zum Mitsingen einladenden Melodien umgesetzt werden (»Heute trinken wir richtig«, 1985; »Dick und durstig«, 1987; »Kneipenterroristen«, 1988). Sex ist wie bei Westernhagen ein von Gefühlen abgekoppelter Trieb (»Mädchen«, 1984), die Jagd nach weiblichen Trophäen erscheint eher als drogeninduzierter Unfall (»Von Glas zu Glas«, 1987) denn als vermeintlich klassenkämpferischer und rebellischer männlicher Akt.

Die homosoziale Einheit Band, die sich anfangs selbst genügt, wird sehr schnell über die Fans zur Familie erweitert, die man, so die Inszenierung (vgl. Hartsch 1997: 9-41), im realen Leben nie hatte. Keine mir bekannte Band singt so viele Loblieder auf sich selbst und ihre Fans wie die Böhsen Onkelz. Mag die Motivation in den Anfangszeiten noch gewesen sein, sich selbst von der eigenen Wichtigkeit zu überzeugen, so gewinnen die entsprechenden Lieder in der Familienkonstruktion ein (neues) Eigenleben. Der Gestern betitelte Teil der aus drei CDs zusammengesetzten Best of-Veröffentlichung Gestern war heute noch morgen (2001) besteht laut Aussage der Band aus Fanfavoriten. Mindestens zehn der siebzehn Titel feiern die homosoziale Einheit von Band und Fans als eine von der Gesellschaft ausgegrenzte. Tabelle 1 (nächste Seite oben) zeigt die auf der CD Gestern enthaltenen Lieder, die Preislieder sind fettgedruckt.

Die homosoziale Einheit von Band und Fans wird zur Parallelgesellschaft, die bietet, was die Gesellschaft vermeintlich verwehrt: Anerkennung und $\mathrm{Si}$ cherheit, verstanden als Bereitstellung eines festen Platzes innerhalb einer bestehenden Ordnung: »Mit dieser Band hast du nicht viele Freunde. Doch die, die du hast, teilen deine Träume. Die, die du hast, teilen alles mit dir« (»Danket dem Herrn«, 1996). Gleichzeitig wird schon die Mitgliedschaft in der Parallelgesellschaft zum hinreichenden Marker für die rebellische Grundhaltung, die der von Theo Gromberg nicht unähnlich ist, aber bei weitem nicht so stark individualisiert und aus Klassenbezügen gelöst erscheint. Im Gegensatz zu Westernhagen, dessen Theo über seine schwächliche Statur trotz allem sympathisch wirken will, wollen die Böhsen Onkelz auch nicht 


\begin{tabular}{|c|c|c|c|}
\hline Nr. & Titel & Album & Jahr \\
\hline 1 & $\gg$ Dunkler Ort« & $\begin{array}{l}\text { Ein böses Märchen... Aus } \\
\text { tausend finstren Nächten }\end{array}$ & 2000 \\
\hline 2 & $\gg$ Finde die Wahrheit« & Hier sind die Onkelz & 1995 \\
\hline 3 & »Kirche « & E.I.N.S. & 1996 \\
\hline 4 & »Nichts ist für die Ewigkeit« & Es ist soweit & 1990 \\
\hline 5 & $\gg$ Onkelz 2000« & $\begin{array}{l}\text { Ein böses Märchen...Aus tausend } \\
\text { finstren Nächten }\end{array}$ & 2000 \\
\hline 6 & $\gg$ So sind wir« & Kneipenterroristen & 1988 \\
\hline 7 & $»$ Terpentin « & Viva los Tioz & 1998 \\
\hline 8 & »Wir ham' noch lange nicht genug« & Wir ham' noch lange nicht genug & 1991 \\
\hline 9 & »Hier sind die Onkelz« & Hier sind die Onkelz & 1995 \\
\hline 10 & »Auf gute Freunde« & E.I.N.S. & 1996 \\
\hline 11 & »Danket dem Herrn« & E.I.N.S. & 1996 \\
\hline 12 & »Danke für nichts « & Hier sind die Onkelz & 1995 \\
\hline 13 & »Lieber stehend sterben« & Weiß & 1993 \\
\hline 14 & $\gg$ Nur die Besten sterben jung « & Wir ham' noch lange nicht genug & 1991 \\
\hline 15 & »Kneipenterroristen« & Kneipenterroristen & 1988 \\
\hline 16 & $\gg$ Nie wieder $\ll$ & Kneipenterroristen & 1988 \\
\hline 17 & $\gg$ Das ist mein Leben $«$ & Wir ham' noch lange nicht genug & 1991 \\
\hline
\end{tabular}

Tabelle 1: Böhse Onkelz - Gestern (2001), Trackliste

gefallen, sie leben von der Inszenierung des Ausgegrenztseins. ${ }^{19}$

Elemente von Individualisierung finden sich jedoch ebenfalls bereits in den 1980er Jahren, also weit vor dem kommerziellen Erfolg der Band. So mündet die Powerballade »Erinnerungen « (1987) in die Erkenntnis, dass der Protagonist seinen Weg allein gehen muss. Er verabschiedet sich aus der Männerhorde. Damit passt die Band sukzessive das von ihr propagierte Männerbild an die von Meuser (2012) benannten Anforderungen der Gegenwart an. Der Familienzusammenhang wird jetzt als temporäre Vereinigung von Individuen gedacht, die ihren Weg jeweils allein gehen können müssen. Hier sind auch deutliche Parallelen zur den Böhsen Onkelz nahestehenden Hooliganszene auszumachen, deren individualisierte Struktur nach Matthesius (1992: 81-110) aus der Auflösung verbindlich strukturierter proletarischer Fangemeinschaften entsteht.

Die Ausdifferenzierung unterschiedlicher Männlichkeiten zeigt sich bei den Böhsen Onkelz im Spannungsfeld von marginalisierter und komplizenhafter Männlichkeit und bildet entsprechende Teilhierarchien heraus. Das

19 Das auf der DVD La Ultima/Live in Berlin (2005) festgehaltene Ende der Abschiedstournee zelebriert nochmals die Familiengemeinschaft in einer abschlieBenden 8-minütigen Sequenz. Bereits während des gesamten Konzertes sind projizierte Bilder ausgewählter Fans Teil der Inszenierung. 
Image der Gruppe basiert auf einem hegemonialen starken Rebellen, Bassist und Texter Weidner, und einem marginalisierten schwachen Rebellen, Sänger Russell, der ständig in Gefahr steht, den Kampf gegen die eigenen Dämonen zu verlieren. Die starke Männerfigur treibt die Individualisierung voran, die schwache fühlt sich durch diese Individualisierung bedroht. Das Bild wird durch zwei ehrliche Arbeiter komplettiert, Gitarrist Röhr und Schlagzeuger Schorowsky. Gleichwohl ist in der Realität Gitarrist und Komponist Röhr ein wichtiger Faktor der musikalischen Entwicklung und Individualisierung der Männlichkeit, während Schlagzeuger Schorowsky den Ruhepol gibt (vgl. Hartsch 1997).

Grundlage der männlichen Inszenierung der Onkelz ist dabei Authentizität. Während Westernhagen eine Rolle spielt und dies auch in seiner filmischen Autobiografie (Rossacher 2010) betont, wählen die Onkelz den gegenteiligen Weg: Persona, Performer und Charakter erscheinen bei Ihnen als identisch.

»Denn wir sind anders, stolz und unbequem. [...] Ich will lieber stehend sterben, als kniend leben, lieber tausend Qualen leiden, als einmal aufzugeben « (»Lieber stehend sterben«, 1993). »Wir« und »ich« sind Synonyme für die Band und ihre Fans. Die Böhsen Onkelz laden zur Identifizierung ein und bieten sich als Spiegelbild an. Die als Gegner ausgesuchten Gesellschafts- respektive Medienvertreter bleiben dabei nicht zwingend anonym, sondern können auch direkt angesprochen werden. Allerdings liegt der pathetische Fokus immer auf der Versicherung der eigenen Stärke und Unbeugsamkeit:

»Wir feiern uns solange es uns gibt,

Auch wenn nicht jeder Arsch uns liebt.

Gepriesen sei der Name dieser Band.

Betet zu Gott, dass ihr uns kennt.

Manchmal ist es ganz schön hart,

Doch jede eurer Lügen macht uns stark«

(Böhse Onkelz: »Gehasst verdammt vergöttert«, 1992).

Diese männliche identitäre Inszenierung durchzieht auch die autorisierte Biografie der Onkelz, Danke für Nichts (Hartsch 1997). Langjährige Paarbeziehungen werden beispielsweise als Grund angeführt, weshalb Gitarrist Röhr und Schlagzeuger Schorowsky eher selten Erwähnung in der Biografie finden. Diese basiert vielmehr auf der Yin Yang-Inszenierung der beiden Hauptfiguren Russell und Weidner. Deren jeweilige Liebesbeziehungen finden zwar Platz, werden aber zumindest im Fall von Weidner so weit wie möglich von der homosozialen Bandfamilie fern gehalten. Sänger Russell 
verkörpert dabei im Bandkontext Figuren, die Texter Weidner entwirft, diese sollen aber laut Biografie wiederum grundsätzlich auf autobiografisch geprägten Erlebnissen und Beobachtungen beruhen, das heißt die Texte inszenieren Weidners Wahrnehmung von Russell. Die Dichotomie von Stärke und Schwäche wird so nochmals zementiert. Texter und Bassist Weidner nimmt auch auf der Bühne die Rolle des Starken ein. Er übernimmt die Kommunikation mit dem Publikum, Sänger Russell ergänzt höchstens kurze Einwürfe, Gitarrist und Röhr und Schlagzeuger Schorowsky bleiben mehrheitlich stumm.

Der musikalische Authentizitätsmarker, der den Idiolekt der Böhsen Onkelz prägt, ist dementsprechend der Stimmklang von Sänger Russell, der als »eure Stimme aus der Gosse« (»Danket dem Herrn«, 1996) vermarktet wird. Russells eigentlich klare Tenorstimme ist mit gutturalen Elementen angereichert, die ein sofort wiedererkennbares Timbre ergibt. Er könnte zwar auch schön singen, aber die schwer erträgliche Realität verzerrt seine Stimme (vor Wut) und lässt ihn (vor Schmerz) schreien, so (m)eine Deutung. In melodischer Hinsicht doppelt sich dies in erzählenden Strophen mit eher statischer Melodik und hymnenartigen Chorusmelodien, die grundsätzlich leicht mitsingbar sind und von Weidner stimmlich gedoppelt werden. Gleichzeitig entwickeln die Böhsen Onkelz ihren Powerchord basierten Punkrock in Richtung Hard und Mainstream Rock weiter, in den sie auch Details anderer musikalischer Stilrichtungen von Metal bis hin zu elektronischer Tanzmusik integrieren, mehrheitlich aber bei einfachen Vers-Chorus-Strukturen verharren. Die Produktionen sind dabei generell um klangliche Aktualität bemüht. Strukturell und klanglich machen die Böhsen Onkelz mit ihrer im Laufe der Karriere immer professioneller inszenierten also ein breites Identifikationsangebot.

Ein zentrales Motiv in den Texten, die Weidner für Russell schreibt, ist Schmerz. ${ }^{20}$ Dabei ist das Erleiden von Schmerz sowohl Notwendigkeit für die Erhaltung der Familie (»Lieber stehend sterben«, 1993), zu zahlender Preis für die Exzesse als auch mit Lust besetzt. Songtitel wie »Bin ich nur glücklich, wenn es schmerzt (1998) und Refrains wie »Komm schon, lass uns leiden, lass uns träumen gehen« (»Leiden«, 1990) sprechen eine deutliche Sprache. Die Lust am Schmerz unterscheidet die Männerfiguren der Böhsen Onkelz deutlich von der Westernhagenschen Weigerung Schmerz zuzulassen (»Dass du mich verlässt, das tut mir nicht weh«, 1980). Zwar erleidet auch Westernhagens Theo Gromberg Schmerz - aber als notwendiges Übel für seine Weigerung Kräfteverhältnisse realistisch einzuschätzen. Die Lust am

20 Die Böhse Onkelz-Vorbilder Rose Tattoo betiteln ein Album Pain (2002). 
Schmerz sublimiert bei den Böhsen Onkelz den Verlust an Bindungen, den die Individualisierung mit sich bringt. In David Savrans (1998: 27-38) an Sigmund Freud angelehnter Terminologie modernisiert sich bei den Böhsen Onkelz der christliche Masochismus des gegenkulturellen Mannes zu Zeiten von Easy Rider, der sein feminiertes Sein einem höheren Zweck unterordnet, zum selbstreflexiven Sadomasochismus von John Rambo, der über seine permanente Selbstfolter durch eine reale Verweiblichung nicht mehr in seiner Männlichkeit bedroht ist (vgl. Elflein 2011: 83f.). Die starke und die schwache Männerfigur der Onkelz repräsentieren diese unterschiedlichen Männlichkeiten, während Westernhagen auf der Ebene des christlichen Masochismus verbleibt. BDSM als präferierte sexuelle Variante findet sich bei den Männerfiguren der Böhsen Onkelz sowohl direkt (»Vier Dominas für mich allein, so soll es sein « heißt es in »Könige für einen Tag«, 1989) als auch verklausuliert (»Lack und Leder«, 1988). Eines der ersten Liebeslieder der Onkelz (»Keine ist wie du«, 1990) richtet sich auch nicht an eine Frau, sondern an die erste Tätowierung, also an etwas, das man sich zufügt, um sowohl den Schmerz des Ausgegrenztseins als auch die Zugehörigkeit zu einer Gruppe zu dokumentieren sowie die Körpergrenzen zu definieren. ${ }^{21}$

Der Exzess als temporäres Vergessen des Schmerzes verliert bei den Böhsen Onkelz in den 1990er Jahren zunehmend an Bedeutung. Die Zeile »Lieder gegen Schmerz «, die das Titelstück von Heilige Lieder (1992) abschließt, ist weniger Selbstbestätigung als eine finale Hommage an die eigene Vergangenheit. Die erfolgreiche Bekämpfung des männlichen Schmerzes lässt sich ab jetzt vielmehr in der Zeile »Ich muss meinen Weg alleine gehen« (»Erinnerungen«, 1987) zusammenfassen. Dieser allein zu gehende Weg führt weg, wahlweise an einen anderen geografischen oder als Metapher an einen anderen psychischen Ort der Selbsterkenntnis und Selbsterfahrung. ${ }^{22}$ »Kann ich mich finden, indem ich mich verlier?« (»1000 Fragen«, 1993b) stellt die alten Lösungswege infrage. Die Zeilen »denn bevor man andere lieben kann, liebe erstmal dich« (»Das Wunder der Persönlichkeit«, 1993a) und »wenn ihr etwas ändern wollt, fangt bei euch an« (»Entfache dieses Feuer«, 1993a) zeigen die neue Marschrichtung. Alle drei Zitate stammen nicht zufällig von den auf den kommerziellen Durchbruch mit

21 Zum Bedeutungswandel von Tätowierungen vgl. Lobstädt 2011. Russells Tätigkeit als Tätowierer findet in der Autobiografie immer wieder großen Raum in Wort und Bild (vgl. exemplarisch Hartsch 1997: 115, 162ff.).

22 Die Autobiografie thematisiert dies über eine prägend und verändernd wirkende Mexikoreise von Bassist und Texter Weidner (vgl. Hartsch 1997: 179f.). Gitarrist Röhr wandert nach der Trennung der Böhsen Onkelz nach Uruguay aus (vgl. Berg 2011). 
Heilige Lieder (1992) folgenden Veröffentlichungen Weiß (1993a) und Schwarz (1993b).

Die in Tabelle 2 fettgedruckten Lieder des Schmerzes und des Aufbruchs bilden denn auch einen Kern der bereits erwähnten Best of-CD-Box Gestern war heute noch morgen (2001), nämlich den Schwerpunkt der aus Bandfavoriten bestehenden zweiten CD Heute.

\begin{tabular}{|c|c|c|c|}
\hline Nr. & Titel & Album & Jahr \\
\hline 1 & »Das Wunder der Persönlichkeit« & Weiß & 1993 \\
\hline 2 & $\gg \mid c h \ll$ & Hier sind die Onkelz & 1995 \\
\hline 3 & »Für immer« & Weiß & 1993 \\
\hline 4 & »Du kannst alles haben« & Hier sind die Onkelz & 1995 \\
\hline 5 & »Ein langer Weg« & Heilige Lieder & 1992 \\
\hline 6 & »Entfache dieses Feuer« & Weiß & 1993 \\
\hline 7 & »Schutzgeist der Scheiße« & $\begin{array}{l}\text { Ein böses Märchen... Aus } \\
\text { tausend finstren Nächten }\end{array}$ & 2000 \\
\hline 8 & »Worte der Freiheit« & Schwarz & 1993 \\
\hline 9 & »Koma - eine Nacht die niemals endet« & E.I.N.S. & 1996 \\
\hline 10 & »Deutschland im Herbst « & Weiß & 1993 \\
\hline 11 & $\gg$ Regen« & E.I.N.S. & 1996 \\
\hline 12 & $\gg 1000$ Fragen « & Schwarz & 1993 \\
\hline 13 & »Bin ich nur glücklich wenn es schmerzt« & Viva los Tioz & 1998 \\
\hline 14 & $\gg$ Weit weg« & Viva los Tioz & 1998 \\
\hline 15 & »Der Schrei nach Freiheit« & Heilige Lieder & 1992 \\
\hline
\end{tabular}

Tabelle 2: Böhse Onkelz - Heute (2001), Trackliste

In diesem Zusammenhang wird die angedeutete Dichotomie von Texter Weidner und Sänger Russell neu bedeutsam. Russell mutiert in der Inszenierung vom Sprachrohr zum Problemkind, das es zu schützen und zu integrieren gilt. Ein Herzstück der Biografie (Hartsch 1997) ist die Authentifizierung zweier gegensätzlicher Männerfiguren, die in Savrans Modell die zwei Seiten einer Medaille, der reflexiv sadomasochistischen Prägung des modernen Mannes darstellen. Die Alternative des Schwachen ist und bleibt der Drogenexzess, der zum Synonym für Schwäche wird, wenn er nicht mehr auf den Feierabendexzess begrenzbar bleibt, also zur Sucht wird (»Hast du Sehnsucht nach der Nadel«, 1990). Die hierarchische Familienstruktur der Böhsen Onkelz tritt in Persona Weidner an, diese Probleme als persönliches und unternehmerisches Projekt in den Griff zu kriegen. Gleichzeitig benötigt das Unternehmen Böhse Onkelz die Männerfigur Russell für seinen Erfolg. Weidner und Russell verkörpern unterschiedliche Facetten der für das Image und den Erfolg der Böhsen Onkelz benötigten Authentizität. 
Die Auflösung fester gesellschaftlicher Strukturen, die Individualisierungsschübe des neoliberalen Kapitalismus führen die Männer der Onkelz zu temporär exzessivem Verhalten, sei es Drogengenuss oder Gewalt. Die schwachen Männer führt der Exzess in die Sucht und/oder ins Gefängnis, die starken werden zu Unternehmern ihrer selbst. Dabei fangen die Starken die Schwachen in der propagierten Familienstruktur auf. Als diese Inszenierung nicht mehr aufrecht erhalten werden kann, lösen sich die Onkelz auf. Als Grund wird posthum die zum wiederholten Male eskalierende Drogensucht von Sänger Russell genannt. Aus dem gleichen Grund muss die überwundene Sucht von Russell sowie seine Läuterung im Gefängnis als Begründung für die im Moment der Niederschrift dieses Artikels in der Presse angekündigte und mit einem Exklusivinterview (Weckmann 2014) medial unterfütterte Wiedervereinigung der Band dienen.

\section{Fazit}

Die Repräsentationen von Männlichkeit bei Westernhagen und den Böhsen Onkelz transformieren angelsächsische (sub-)proletarische und gleichzeitig spätkapitalistisch individualisierte Männerbilder in die deutschsprachige populäre Musik, auch um die sprachliche Begrenzung ihres Marktes nicht als künstlerische begreifen zu müssen. Westernhagen arbeitet in der Folge der besprochenen Theo-Trilogie verstärkt mit internationalen Musikern und verwirklicht sich mit der Realisierung seines in New York aufgenommenen und einen Stadtteil von Brooklyn zum Titel machenden Spätwerks Williamsburg (2009) einen Traum (vgl. Rossacher 2010). Auch die Böhsen Onkelz inszenieren den deutschen Markt schnell als Begrenzung. »Alles was die Onkelz ausmachte, orientierte sich an internationalen Maßstäben, fernab von der einheimischen Provinzialität der 80er Jahre« (Röhr/Larmann 2006: 5).

Die Männerfiguren, die Westernhagen als Theo inszeniert, orientieren sich vornehmlich an den USA und lassen proletarisches Bewusstsein weitgehend vermissen. Sie sind symbolisch überzeichnete und zum Scheitern verurteilte Zerrbilder des hegemonialen Mannes als erfolgreicher Unternehmer. Der Westernhagensche Mann verliert in seinem Kampf um Anerkennung die positiven Elemente seiner Herkunft, gewinnt aber kaum neue hinzu. Aus dieser Tragik schlägt er als romantischer Antiheld Gewinn und mutiert zur sympathischen Unterhaltungsfigur, zum erzwungenen Entertainer seiner selbst. Gleichwohl ist er in seinem grenzenlosen Egoismus, der sich als u.a. als Sexismus verwirklicht, ein Vorbote des Neoliberalismus. 
Die Böhsen Onkelz orientieren sich an stärker in der Arbeiterklasse verwurzelten angelsächsischen Männerbildern. Aufgrund ihrer geschickten, autobiografisch geerdeten Inszenierung können sie ihr Publikum überzeugen, dass sie im Gegensatz zu Westernhagen keine Rolle spielen, aber dem von Westernhagen verkörpertem Milieu entstammen. Ihre Männlichkeit inszeniert sich als marginalisierte mit den dazugehörigen Insignien von Gewalt bis zur Tätowierung. Dementsprechend wird das Angebot der Böhsen Onkelz auch medial stärker skandalisiert als Westernhagens Inszenierung. Gleichwohl beruht die Skandalisierung der Böhsen Onkelz auch auf (der Androhung) realer Gewaltausübung gegenüber Schwächeren.

Die männlichen ernsten Spiele des Wettbewerbs werden auf dem Weg von Westernhagen zu den Onkelz verschärft und verlieren das spielerische Element. Massenwirksamkeit erreicht die Band dementsprechend erst, als sie einen Weg aus der symbolischen Überzeichnung marginalisierter Männlichkeit aufzeigt. Im Einklang mit angelsächsischen Idealen wird ein Mythos des hegemonialen Mannes kopiert: Individualisierung wird als Disziplinierung verstanden. Der von den Böhsen Onkelz inszenierte Mann diszipliniert sich zum erfolgreichen Unternehmer seiner selbst. Um die Bindung an die Gruppe über die Individualisierung nicht zu verlieren, wird diese zur Familie umdefiniert, die qua Zugehörigkeit auch die auffängt, die wie Westernhagens Mann scheitern. Damit machen die Böhsen Onkelz ihren Fans auch ein Integrationsangebot in die deutsche Gesellschaft. Bei der Filmfigur Theo bleibt diese Rolle seinem italienischen Sidekick vorbehalten, der musikalischen Transformation fehlt diese Ebene. Der Musiker Westernhagen kennt Ende der 1970er Jahre noch keinen Ausweg aus seinem Dilemma, er lebt seine Träume als exzessives Feierabendvergnügen des Arbeiters, die Böhsen Onkelz in den 1990ern vermeintlich schon: Chef eines Familienunternehmens werden.

\section{Literatur}

Bakhtin, Mikhail (1986). Speech Genres and Other Late Essays. Hg. v. Carl Emerson und Michael Holquist. Austin: University of Texas Press.

Barger, Ralph »Sonny« (2003). Hell's Angel. Mein Leben. Reinbeck: Rowohlt.

Berg, Marcus (2011). »Matt Gonzo Roehr im Interview: >Eine Kopie kann nie so gut sein wie das Original! « «In: Pressuremagazine.de, http://www.pressuremaga zine.de/interview/2985/matt-gonzo-roehr-im-interview-eine-kopie-kann-nie-sogut-sein-wie-das-original.html (Zugriff am 17.2.2014).

Claus, Robert / Lehnert, Esther / Müller, Yves (Hg.) (2010). Was ein rechter Mann ist.... Männlichkeiten im Rechtsextremismus. Berlin: Karl Dietz. 
Connell, R.W. (Raewyn) (1995). Masculinities. Berkeley, Los Angeles: University of California Press.

Eberwein, Markus / Drexler, Josef (1987). Skinheads in Deutschland. Interviews. Hannover, München: Selbstverlag.

Ehnert, Günter (1979). Rock in Deutschland. Lexikon deutscher Rockgruppen und Interpreten. Hamburg: Taurus Press.

Elflein, Dietmar (2011). "What Is This That Stands Before Me? - Männerbilder im Heavy Metal.«In: Thema Nr. 1. Sex und populäre Musik (= Beiträge zur Popularmusikforschung 37). Hg. v. Dietrich Helms und Thomas Phleps. Bielefeld: transcript, S. 79-96.

Erhart, Walter (2005). »Das zweite Geschlecht: >Männlichkeit< interdisziplinär, ein Forschungsbericht. « In: Internationales Archiv für Sozialgeschichte der Literatur 30, Nr. 2, S. 156-232.

Fenske, Uta / Schuhen, Gregor (Hg.) (2012). Ambivalente Männlichkeit(en). Maskulinitätsdiskurse aus interdisziplinärer Perspektive. Opladen, Berlin, Toronto: Barbara Budrich.

Fenske, Uta (2012). »Männlichkeiten im Fokus der Geschlechterforschung. Ein Überblick.«In: Fenske/Schuhen, S. 11-27.

Frith, Simon / Horne, Howard (1987). Art into Pop. London, New York: Routledge.

Frith, Simon (2006). "Why do songs have words?« [1988]. In: Soziale Horizonte von Musik. Ein kommentiertes Lesebuch zur Musiksoziologie. Hg. v. Christian Kaden und Karsten Mackensen. Kassel u.a.: Bärenreiter, S. 157-174.

Gerards, Marion / Loeser, Martin / Losleben, Katrin (Hg.) (2013). Musik und Männlichkeiten in Deutschland seit 1950. Interdisziplinäre Perspektiven (= Beiträge zur Kulturgeschichte der Musik 8). München: Allitera.

Haring, Hermann (1984). Rock aus Deutschland West. Reinbek: Rowohlt.

Hartsch, Edmund (1997). Böhse Onkelz - Danke für nichts. Frankfurt/M.: B.O. Management AG.

Heesch, Florian / Losleben, Katrin (Hg.) (2012). Musik und Gender. Ein Reader. Köln: Böhlau.

Horlacher, Stefan (2013). »Männlichkeitsforschung: Ein einleitender Überblick.« In: Gerards/Loeser/Losleben, S. 23-38.

Kravetz, Jean-Jacques / Drewing, Christina (2008). Meine 40 Jahre in der deutschen Rockmusik. Von Frumpy über Udo Lindenberg bis Peter Maffay. Heidelberg: Palmyra.

Kübler, Olaf (2005). »Marius Müller-Westernhagen.«In: Gerhard Augustin, Der Pate des Krautrock. Berlin: Bosworth, S. 317-325.

Lobstädt, Tobias (2011). Tätowierung, Narzissmus und Theatralität. Selbstwertgewinn durch die Gestaltung des Körpers. Wiesbaden: VS.

Loeser, Martin (2013). »>Wann ist ein Mann ein Mann?< Männlichkeitsinszenierungen in westdeutscher Rock- und Popmusik am Beispiel von Marius Müller-Westernhagen und Herbert Grönemeyer.«In: Gerards/Loeser/Losleben, S. 121-140.

Losleben, Katrin (2013). »Musik und Männlichkeiten - ein Forschungsüberblick. «In: Gerards/Loeser/Losleben, S. 53-69.

Matthesius, Beate (1992). Anti-Sozial-Front. Vom Fußballfan zum Hooligan. Opladen: Leske und Budrich.

Meuser, Michael (2012). »Männlichkeiten in Bewegung. Zur Aktualität des Konzepts hegemonialer Männlichkeit angesichts des Wandels von Erwerbsarbeit.« In: Fenske/Schuhen, S. 147-164. 
Niemczyk, Ralf (2006). »Popsprache Deutsch.«In: Goethe Institut, Die Macht der Sprache, http://www.goethe.de/lhr/prj/mac/spw/de1396844.htm (Zugriff am 12.2.2014).

Poole, Ralph J. (2012). Gefährliche Maskulinitäten. Männlichkeit und Subversion am Rande der Kulturen. Bielefeld: transcript.

Projektgruppe Mannopoly (Hg.) (2012). Kerle Kulte. Inszenierungen von Männlichkeit. Berlin: Archiv der Jugendkulturen.

Rauhut, Michael (1993). Beat in der Grauzone. DDR-Rock 1964 bis 1972 - Politik und Alltag. Berlin: BasisDruck.

Rauhut, Michael (1996). Schalmei und Lederjacke. Udo Lindenberg, BAP, Underground - Rock und Politik in den achtziger Jahren. Berlin: Schwarzkopf \& Schwarzkopf.

Röhr, Matthias >Gonzo / Larmann, Ralph (2006). Meine letzten 48 Stunden mit den Böhsen Onkelz. Berlin: I.P. Verlag Jeske/Mader.

Savran, David (1998). Taking It Like a Man. White Masculinity, Masochism, and Contemporary American Culture. Princeton, NJ: Princeton University Press.

Simmons, David (2008). The Anti-Hero in the American Novel. From Joseph Heller to Kurt Vonnegut. New York u.a.: Palgrave Macmillan.

Virchow, Fabian (2010). "Tapfer, stolz, opferbereit. Überlegungen zum extrem rechten Verständnis >idealer Männlichkeit<.«In: Was ein rechter Mann ist... Männlichkeiten im Rechtsextremismus. Hg. v. Robert Claus, Esther Lehnert und Yves Müller. Berlin: Karl Dietz, S. 39-52.

Walser, Robert (2012). »Running with the Devil. Macht Geschlecht und Wahnsinn im Heavy Metal« [1993]. In: Heesch/Losleben, S. 272-286.

Weckmann, Matthias (2010). »Blutsbrüder.« In: Rollingstone.de, http://www. rollingstone.de/das-archiv/article250227/print/blutsbrueder.html (Zugriff am 17.2.2014).

Weckmann, Matthias (2014). »Böhse Onkelz: Nichts ist für die Ewigkeit.«In: Metal Hammer, H. 3 (März), S. 20-29.

Wicke, Peter / Müller, Lothar (Hg.) (1996). Rockmusik und Politik. Analysen, Interviews und Dokumente. Berlin: Ch. Links.

Wicke, Peter / Ziegenrücker, Kai-Erik / Ziegenrücker, Wieland (2004). Lexikon der populären Musik. DVD. Mainz: Schott.

\section{Diskographie}

Böhse Onkelz (1984). »Mädchen.« Auf: Der nette Mann. Rock-o-Rama RR 40.

Böhse Onkelz (1985). »Heute trinken wir richtig. « Auf: Böse Menschen Böse Lieder. Rock-o-Rama RRR 48.

Böhse Onkelz (1987). »Dick und durstig «, »Erinnerungen«, »Von Glas zu Glas.« Auf: Onkelz wie wir... Metal Enterprises ME 507.

Böhse Onkelz (1988). »Kneipenterroristen«, »Lack und Leder.« Auf: Kneipenterroristen. Metal Enterprises ME 519.

Böhse Onkelz (1989). »Könige für einen Tag.« Auf: Lügenmarsch. Metal Enterprises ME 530.

Böhse Onkelz (1990). »Hast du Sehnsucht nach der Nadel«, »Keine ist wie du«, »Leiden. « Auf: Es ist soweit. Metal Enterprises ME 555. 
Böhse Onkelz (1992). »Gehasst, verdammt, vergöttert«, »Heilige Lieder.« Auf: Heilige Lieder. Bacillus Records/Bellaphon 290-09-072.

Böhse Onkelz (1993a). »Das Wunder der Persönlichkeit, »Entfache dieses Feuer «, »Lieber stehend sterben. « Auf: Weiß. Bacillus Records/Bellaphon 290-09-073.

Böhse Onkelz (1993b). »1000 Fragen. " Auf: Schwarz. Bacillus Records/Bellaphon 290-09-074.

Böhse Onkelz (1996). »Danket dem Herrn.« Auf: E.I.N.S. Onkelz Prod./Virgin 72438 4234928.

Böhse Onkelz (1998). »Bin ich nur glücklich, wenn es schmerzt.« Auf: Viva los Tioz. Onkelz Prod./Virgin 724384652425.

Böhse Onkelz (2001). Gestern war heute noch morgen. 3 CDs. Rule23 Recordings/ Virgin CD BOX 8100052.

Deutscher, Drafi And His Magics (1965). Marmor Stein und Eisen bricht. 7". Decca D 19735.

Die Yankees (1965). »Halbstark«. 7". Polydor 52589.

Ihre Kinder (1969). Ihre Kinder. Philips 844393 PY.

Lindenberg, Udo \& das Panikorchester (1974). »Rudi Ratlos. A Auf: Ball Pompös. Telefunken SLE 14790-P.

Lindenberg, Udo \& das Panikorchester (1976). »Bodo Ballermann. Auf: Galaxo Gang. Telefunken 6.22460 AS.

Maffay, Peter (1979). Steppenwolf. Telefunken 6.23777.

Müller-Westernhagen, Marius (1977). "Träumer.« Auf: Ganz allein krieg ich's nicht hin. Warner Brothers WB 56381.

Müller-Westernhagen, Marius (1978). »Dicke«, »Johnny Walker«, »Mit 18«, Mit Pfefferminz bin ich dein Prinz «, »Zieh dir bloß die Schuhe aus. « Auf: Mit Pfefferminz bin ich dein Prinz. Warner Brothers WB 56567.

Müller-Westernhagen, Marius (1980). „Der Junge auf dem weißen Pferd«, »Hass' mich oder lieb' mich«, Lady«, Mein Schatz«, »Sie hat meine Lunte gefunden.« Auf: Sekt oder Selters. Warner Brothers WB 56792.

Müller-Westernhagen, Marius (1981). »Daß du mich verläßt«, »Ein Loch in der Tasche«, »Lulu.« auf: Stinker. Warner Brothers WB 56906.

Rose Tattoo (1980). »Nice Boys. « Auf: Rock'n'Roll Outlaw. WEA 58144.

Rose Tattoo (2002). »Union Man. « Auf: Pain. Steamhammer/SPV 085-74212 CD.

Sparifankal (1976). Bayern Rock. April 0000.

V.A. (2000). Das war ein harter Tag. Beatles Lieder auf Deutsch. Bear Family Records BCD 15915.

West, Marius (1974). »Celebration«. 7". Polydor 2041519.

Westernhagen (2009). Williamsburg. Kunstflug KUNST003.

\section{Filmographie}

Benedek, Laszlo (Regie) (1953). The Wild One. Sony Home Entertainment B00 004RYPV, 1999.

Böhse Onkelz (2005). La Ultima/Live in Berlin. Rule23 Recordings/Tonpool 565390197.

Bringmann, Peter F. (Regie) (1977). Aufforderung zum Tanz. WDR/Pidax B00 91SNU8Q, 2012. 
Bringmann, Peter F. (Regie) (1980). Theo gegen den Rest der Welt. Edition Deutscher Film/Studio Canal B0002H3UBC, 2004.

Burns, Kevin (Regie) (2010). Star Wars. Die Geschichte einer Saga. Arte F.

Hopper, Dennis (Regie) (1969). Easy Rider. Sony Home Entertainment B00004RYUU, 2000.

Klick, Ronald (Regie) (1974). Supermarkt. Filmgalerie 451 3941540556, 2005.

Lemke, Klaus (Regie) (1974). Paul. Hurricane, Hanseplatte, 2008.

Müller-Westernhagen, Marius (1983). In Concert. Magic Music GmbH 3011.

Rossacher, Hannes (Regie) (2010). Marius Müller-Westernhagen. Zwischen den Zeilen. Eine Biografie. Kunstflug Entertainment GmbH B0041G2YR6.

Spils, May (Regie) (1968). Zur Sache, Schätzchen. Schamoni Film und Medien GmbH/Ascot B00DIR8B4Y, 2013.

Whalley, Ben (Regie) (2009). Kraut Rock - The Rebirth of Germany. BBC.

\begin{abstract}
This paper discusses representations of masculinity within Deutschrock - that is, (West-)German rock music with lyrics in German language - using genderorientated narratology. Deutschrock developed in two waves, the first one, prominent in the 1970s, is based on a left wing consciousness moving to political centre ground, while the second one begins after the millennium and moves from the political right to centre ground. The article discusses Marius Müller-Westernhagen as representative of the first wave and Die Böhsen Onkelz representing the second wave. It is argued, that both perform masculinities that are rooted in an Anglo-Saxon male proletarian rebel figure. Both transform this male figure in a specific way. On its way from the first to the second wave of Deutschrock the male figure gets modernised. In terms of Reawyn W. Connell Westernhagen performs a distorted figure of hegemonic masculinity while the performance of die Böhsen Onkelz represents marginalised masculinity. In David Savran's terminology Westernhagen's male representations are still rooted in Christian masochism while the male representations of Die Böhsen Onkelz divide self-reflexive sadomasochism between two performers.
\end{abstract}

